\title{
Structural and Functional Properties of an Upper Airway Dilator Muscle in Aged Obese Male Rats
}

\author{
J. Richard Skelly Ruth A. O'Connell James F.X. Jones Ken D. O'Halloran \\ UCD School of Medicine and Medical Science, Health Sciences Centre, University College Dublin, Dublin, Ireland
}

\section{Key Words}

Antioxidants $\cdot$ Fatigue $\cdot$ Hypoxia $\cdot$ Muscle $\cdot$ Obesity $\cdot$

Oxidative stress $\cdot$ Sleep apnoea $\cdot$ Upper airway

\begin{abstract}
Background: Age, obesity and male sex are risk factors for the development of obstructive sleep apnoea syndrome. Objective: We examined structural and functional properties of the sternohyoid muscle in young lean and aged obese male rats. We hypothesized that the aged muscle would be vulnerable to oxidative stress (hypoxia). Methods: Isometric contractile and endurance properties of the sternohyoid muscle were assessed in vitro with or without the superoxide scavenger Tempol (10 mM). Muscle fibre size and density were determined by myosin heavy chain immunofluorescence. Succinate dehydrogenase (SDH) and glycerol-3- phosphate dehydrogenase (GPDH) enzyme activities were determined. Results: Fibre hypertrophy, increased fast twitch (type 2X) fibre density, decreased SDH activity and increased GPDH activity, together with increased force and fatigue, were observed in aged obese muscles compared to young lean muscles. Tempol treatment increased strength and sensitivity to stimulation. Hypoxic depression of force was ameliorated by antioxidant treatment with equivalent effects in
\end{abstract}

young lean and aged obese muscle. Conclusions: We conclude that the rat sternohyoid exhibits indefinite growth and is protected from oxidative stress as the animal ages.

Copyright $\odot 2011$ S. Karger AG, Basel

\section{Introduction}

As rats develop and age they increase in size and weight. Ecologists and zoologists regard the rat as an interparous species with features of indeterminate growth. The size the animal attains when reproductively mature is much less than the ultimate size found in old age. Even old rats still show growth plates in their long bones [1], although longitudinal growth becomes somewhat curtailed by unknown mechanisms. When given free access to food, rats also become obese with age. The combination of obesity and old age is of interest to respiratory physiologists because these represent significant risk factors for development of obstructive sleep apnoea syndrome (OSAS) [2, 3]. Retropharyngeal fat pads may predispose to airway obstruction, and aging of airway musculature may weaken their 'splinting' action on the upper respiratory tract. Previous studies on the aged Fisher rat [4] and the Zucker fatty rat $[5,6]$ have examined these

\section{KARGER}

Fax +4161306 1234

E-Mail karger@karger.ch

www.karger.com
C 2011 S. Karger AG, Basel

$0025-7931 / 11 / 0826-0539 \$ 38.00 / 0$

Accessible online at:

www.karger.com/res
Ken D. O'Halloran, $\mathrm{PhD}$

UCD School of Medicine and Medical Science

Health Sciences Centre, University College Dublin

Belfield, Dublin 4 (Ireland)

Tel. +353 1716 6620, E-Mail ken.ohalloran@ucd.ie 
factors in isolation and found no effect on peak force production, but the combination of these factors may overwhelm the working muscle. Therefore, we decided to study the structure and function of an upper airway dilator muscle (sternohyoid) in young lean and old obese male rats. In addition, we imposed an oxidative stress on the muscle (hypoxia) and manipulated the abundance of reactive oxygen species (ROS) using a superoxide dismutase mimetic (Tempol). We hypothesized that the sternohyoid from old obese rats, despite its indeterminate growth pattern, would exhibit diminished performance especially when subjected to oxidative stress.

\section{Materials and Methods}

\section{Animals}

Experiments were performed on 16 young lean (2-3 months, $309 \pm 18 \mathrm{~g})$ and 16 aged naturally obese (18-20 months, $906 \pm$ $33 \mathrm{~g}$ ) male Wistar rats (Charles River Laboratories, UK). Both groups were fed standard rat chow ad libitum. A qualitative assessment clearly indicated that aged animals had increased adiposity compared to lean controls. All procedures were approved by the Animal Research Ethics Committee, University College Dublin.

\section{In vitro Muscle Preparation}

The animals were anaesthetized with 5\% isoflurane and euthanized by cervical spinal cord transection. The paired sternohyoid muscles were separated along their medial borders and excised. The muscles were placed in a tissue bath at room temperature containing continuously gassed $\left(95 \% \mathrm{O}_{2} / 5 \% \mathrm{CO}_{2}\right)$ Krebs solution. The solution contained the following components: $\mathrm{NaCl}$ $120 \mathrm{mM}$, KCl $5 \mathrm{mM}, \mathrm{Ca}^{2+}$ gluconate $2.5 \mathrm{mM}, \mathrm{MgSO}_{4} 1.2 \mathrm{~mm}$, $\mathrm{NaH}_{2} \mathrm{PO}_{4} 1.2 \mathrm{mM}, \mathrm{NaHCO}_{3} 25 \mathrm{mM}$, glucose $11.5 \mathrm{mM}$ and d-tubocurarine $25 \mu \mathrm{M}$. Longitudinal strips of muscle from each sternohyoid ( $\sim 1.5 \mathrm{~mm}$ in diameter) were prepared and placed vertically in Plexiglas tissue holders in Krebs solution. Strips were cut to retain the full length of the original whole muscle. The two tissue holders were placed in separate water-jacketed organ baths aerated with either $95 \% \mathrm{O}_{2} / 5 \% \mathrm{CO}_{2}$ for control studies or $95 \% \mathrm{~N}_{2} / 5 \%$ $\mathrm{CO}_{2}$ for hypoxia studies (bath $\mathrm{PO}_{2} \sim 45 \mathrm{~mm} \mathrm{Hg}$ ); both baths were maintained at $35^{\circ} \mathrm{C}$. The neuromuscular paralyzing agent d-tubocurarine $(25 \mu \mathrm{M})$ eliminated activation of intramuscular nerve branches, thus ensuring that isometric force generation was due solely to direct muscle stimulation. The muscle strips were positioned between a pair of platinum plate electrodes, with the base fixed to an immobile hook and the other end tied to an isometric force transducer with non-elastic string. The position of the force transducer could be adjusted by a micropositioner, thus altering the length of the muscle strips. A fixed graduated scale located behind the muscle strip allowed the accurate measurement of muscle length.

\section{Protocol}

The optimum length $\left(\mathrm{L}_{\mathrm{O}}\right.$; muscle length producing the maximal isometric twitch force in response to supra-maximal stimu- lation) was determined by incrementally adjusting the micropositioner between intermittent stimulations. The mean muscle length at the $\mathrm{L}_{\mathrm{O}}$ was $1.9 \pm 0.1 \mathrm{~cm}$ in young lean animals and 2.1 $\pm 0.1 \mathrm{~cm}$ in aged obese animals (mean \pm SEM, $\mathrm{p}<0.01$, Student's unpaired t test). Once determined, the muscle remained at this length for the full protocol. Next, the muscle was allowed a 5-min equilibration period. The single isometric twitch force, contraction time, half-relaxation time, stress-frequency relationship and performance during repeated stimulation were then determined in response to electrical field stimulation delivered via the plate electrodes flanking the tissue connected to a square pulse constant current stimulator (Model S44; Grass Instruments, Quincy, Mass., USA). Data were recorded using a commercial data acquisition system (PowerLab; AD Instruments, UK) and stored for later analysis on a computer. First, a single twitch was elicited (supra-maximal voltage, $1 \mathrm{~ms}$ duration). The twitch force, contraction time (time to peak force) and half-relaxation time (time for peak force to decay by 50\%) were determined. Next, the forcefrequency relationship was determined by sequentially stimulating the muscle strips at 10,20,30,40,60, 80 and $100 \mathrm{~Hz}$ for 300 $\mathrm{ms}$ at each stimulus frequency allowing a 2-min recovery interval between stimuli. Five minutes following the force-frequency protocol, repeated muscle contraction was induced by stimulation at $40 \mathrm{~Hz}$ with $300-\mathrm{ms}$ trains every $2 \mathrm{~s}$ for a period of $2 \mathrm{~min}$. In vitro studies of contractile properties of sternohyoid muscle bundles from young lean and aged obese animals were performed in the absence (control) or presence of 4-hydroxy-2,2,6,6-tetramethylpiperidine-1-oxyl (Tempol, $10 \mathrm{mM})$. Studies were performed under hyperoxic or hypoxic conditions (separate muscle bundles) in parallel. All compounds were purchased from Sigma-Aldrich Company, Dublin, Ireland, and were made up fresh each experimental day.

\section{SDH and GPDH Enzyme Histochemistry}

Longitudinal muscle strips from young lean and aged obese animals were snap-frozen in isopentane, cooled in liquid nitrogen for approximately 10 s and subsequently coated in Tissue-Tek (OCT mounting medium; VWR, Radnor, Pa., USA) before being dipped again in isopentane for a further 20-30 s. Muscles were then stored at $-80^{\circ} \mathrm{C}$ until later use. Serial transverse $10-\mu \mathrm{m}$ sections were cryosectioned (model CM30505; Leica Microsystems, Nussloch, Germany) at $-22^{\circ} \mathrm{C}$ and mounted on polysine-coated glass slides. Muscle sections were stained histochemically for the mitochondrial enzyme succinate dehydrogenase (SDH). SDH activity was determined using an incubation solution containing sodium succinate and nitro blue tetrazolium chloride (NBT) in phosphate buffer brought to $\mathrm{pH} 7.4$ using drops of $\mathrm{NaOH}(1 \mathrm{M})$. The incubation period was $8 \mathrm{~min}$ at $37^{\circ} \mathrm{C}$. Young lean and aged obese muscle sections were processed in parallel. In separate muscle sections, glycerol-3-phosphate dehydrogenase (GPDH) activity was determined using an incubation solution containing DL3 -glycerophosphate (disodium salt), 0.01\% menadione and NBT in phosphate buffer ( $\mathrm{pH}$ 7.4). The incubation period was $20 \mathrm{~min}$ at $37^{\circ} \mathrm{C}$ [7]. Young lean and aged obese muscle sections were processed in parallel. Control (blank) reactions were performed on serial sections using solutions that lacked primary substrate to ensure that non-specific redox reactions did not contribute to the blue histochemical staining. All slides were then dehydrated in a graded series of acetone and methanol rinses. Slides were then passed through xylene and cover-slipped. 


\section{Myosin Heavy Chain Immunohistochemistry}

Indirect immunofluorescence for the myosin heavy chain (MHC) isoform composition was performed on serial unfixed muscle sections. Serial transverse muscle sections (10 $\mu \mathrm{m}$ thick) from young lean and aged obese rats were cryosectioned and placed on polysine-coated glass slides. Sections were incubated for $30 \mathrm{~min}$ in phosphate-buffered solution (PBS), $1 \%$ bovine serum albumin (BSA) and 5\% goat serum to block non-specific binding of antibodies. Sections were incubated with one of two antibody cocktails. Primary antibodies, developed by S. Schiaffino, were obtained from the Developmental Studies Hybridoma Bank (DSHB) developed under the auspices of the NICHD and maintained by University of Iowa Department of Biology, Iowa City, Iowa, USA. One half of the sections in young lean and aged obese rats were incubated with a cocktail of primary antibodies which targeted MHC type 1,2A and 2B fibres. The cocktail consisted of the following mouse anti-myosin antibodies: type 1 IgG2b (1:100), type 2A IgG1 (1:100) and type 2B IgM (1:25). The remaining sections were incubated with a mouse IgG1 primary antibody (1:10) that targeted all but myosin type $2 \mathrm{X}$ fibres. Rabbit anti-laminin antibody (catalogue No. L9393; Sigma-Aldrich) was used to highlight the connective tissue surrounding each fibre. Muscle sections were incubated with the primary antibodies in a humidity chamber overnight at $4{ }^{\circ} \mathrm{C}$. The sections were then washed three times in PBS before application of specific secondary antibodies. For all double-labelled sections, all fibre types apart from type $2 \mathrm{X}$ were highlighted with a Dylight594-conjugated anti-mouse IgG1 secondary antibody (1:500). Laminin labelling was visualized with a FITC-conjugated anti-rabbit secondary antibody (catalogue No. F9887; Sigma-Aldrich). For separate, triple-labelled sections, secondary antibodies included an AlexaFluor350-conjugated goat anti-mouse IgG2b (1:500; Invitrogen), a Dylight594-conjugated goat anti-mouse IgG1 (1:500; Jackson) and an AlexaFluor488-conjugated goat anti-mouse IgM (1:250; Invitrogen). Secondary antibodies were applied for $1 \mathrm{~h}$ in a dark humidity chamber at room temperature. Slides were rinsed in PBS, mounted with Vectashield (H-1000; Vector Laboratories, Peterborough, UK), cover-slipped and stored at $4{ }^{\circ} \mathrm{C}$. Negative control experiments in which the primary antibodies were omitted were also performed.

\section{Lipid Peroxidation Assay}

Thiobarbituric acid-reactive substances (TBARS) were measured using a commercial kit (Cell Biolabs, San Diego, Calif., USA). A mixture of $100 \mu \mathrm{l}$ of muscle homogenate, $100 \mu \mathrm{l}$ SDS lysis solution and $250 \mu \mathrm{l}$ of the manufacturer's TBA colour reagent was incubated in sealed tubes on a heat block at $95^{\circ} \mathrm{C}$ for $50 \mathrm{~min}$. After cooling in an ice bath for $5 \mathrm{~min}$, the mixture was centrifuged at $3,000 \mathrm{~g}$ for $15 \mathrm{~min}$ at $4^{\circ} \mathrm{C}$. The supernatant was mixed with n-butanol and centrifuged at $10,000 \mathrm{~g}$ for $5 \mathrm{~min}$. Absorbance was measured at $532 \mathrm{~nm}$ in a 96-well plate reader (Molecular Devices, Sunnyvale, Calif., USA) and compared with a standard curve constructed with known concentrations of malondialdehyde (MDA). The total protein content in each muscle sample was determined by commercial assay (Thermoscientific, USA) using BSA as the protein standard. Samples were normalized for differences in the amount of muscle protein in each sample and data were expressed as nanomoles of MDA per milligramme of protein.

Preservation of Muscle Force in Old Obese Rats

\section{Data Analysis}

Specific force was calculated in $\mathrm{N} / \mathrm{cm}^{2}$ of muscle cross-sectional area. The latter was approximated by weighing the dry muscle strips at the end of the experimental protocol and dividing this by the product of $\mathrm{L}_{\mathrm{O}}$ and muscle density (assumed to be 1.056 $\left.\mathrm{g} / \mathrm{cm}^{3}\right)$. The force transducers were calibrated using known gramme weights. The contraction time and half-relaxation time were measured as indices of isometric twitch kinetics. For the force-frequency relationship, data were plotted as absolute stress across the range of stimulus frequencies employed in the study. Additionally, non-linear regression analysis was employed (Graph Pad Prism) for control and Tempol groups, allowing us to determine minimum, maximum, slope and $\mathrm{EF}_{50}$ values (i.e. the stimulus frequency producing $50 \%$ of the peak force) for sternohyoid muscle. Finally, to assess muscle performance in response to repeated activation we performed four sets of analyses. First, each tetanic contraction was measured and the specific force was averaged in 10-second bins (i.e. 5 tetanic contractions) over the initial $1 \mathrm{~min}$ and in 30-second bins over the second minute. Second, force potentiation in the early phase of each trial was calculated as follows: [(peak tetanic contraction/initial tetanic contraction $\times 100)-100]$. Third, a fatigue index was calculated as follows: [100 - (final twitch amplitude/initial twitch amplitude) $\times 100]$. Fourth, a performance index was calculated for the whole trial according to the following formula: (average twitch amplitude of all 60 contractions/initial twitch amplitude $\times 100)$ modified from Healy et al. [8]. Values are expressed as means \pm SEM

Statistical comparisons between groups were performed using one-way ANOVA with the Newman-Keuls post hoc test, two-way ANOVA (aged obese $\times$ drug) or three-way ANOVA (aged obese $\times$ drug $X$ frequency or time). Contractile kinetics were compared using a Mann-Whitney test. $\mathrm{p}<0.05$ was considered statistically significant in all tests. To quantify SDH and GPDH enzyme activities, low-power, bright-field images of muscle sections were captured under controlled lighting conditions using a BX51 Olympus microscope (Olympus Life Science Microscopes, Munich, Germany) and an Olympus DP71 camera. The optical density of muscle sections was calculated using Scion Image ${ }^{\mathrm{TM}}$ software (Scion Corporation, Frederick, Md., USA) according to the following equation: $y=\log _{10}[255 /(255-x)]$ for a 256 grayscale image; $x$ equals the gray value of the image. Multiple sections were analyzed per animal and an average optical density was calculated normalized to the total cross-sectional area. All data per animal were first averaged before computing group means. Data are expressed as means \pm SEM. Young lean and aged obese muscles were compared using a two-tailed Student's unpaired t test with $\mathrm{p}<0.05$ considered statistically significant. For immunofluorescence analysis, Cell $\mathrm{A}^{\mathrm{TM}}$ (Olympus) software was used to digitally analyze images and calculate the areal density and cross-sectional area for each MHC fibre type. A stereological method was employed by way of a square test frame $\left(640,000 \mu \mathrm{m}^{2}\right)$ with inclusion and exclusion boundaries randomly placed over muscle sections. Areal density was calculated as the sum of the cross-sectional areas for a given immunolabelled fibre type divided by the area of the square test frame $\times 100$. This approach was considered more physiologically relevant than simple fibre type counts (i.e. numerical density). Values for areal density (\%) and cross-sectional area $\left(\mu \mathrm{m}^{2}\right)$ are expressed as means \pm SEM and were compared across groups by one-way ANOVA with the Newman-Keuls post hoc test, and $\mathrm{p}<0.05$ was considered statistically significant. 


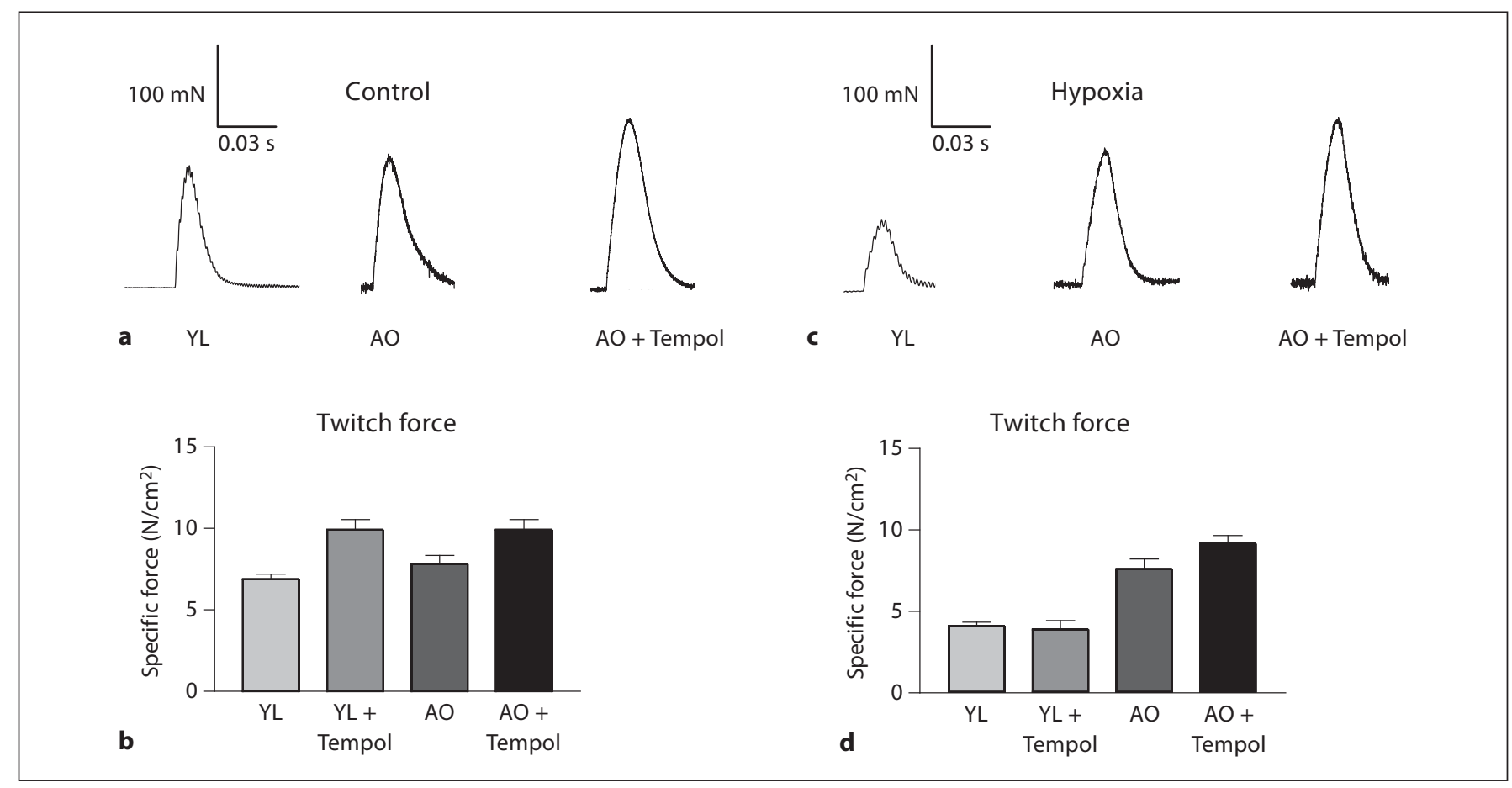

Fig. 1. Twitch. Original traces of isometric single twitch in control (a) and hypoxic (c) conditions. b Values (means \pm SEM) for isometric single twitch force. Both age/obesity and Tempol caused a significant increase in force production. There was no interaction. d Values (means \pm SEM) for the isometric single twitch force in hypoxia. Note the significantly higher specific force recorded in

\section{Results}

\section{Isometric Twitch Force}

Both age/obesity and Tempol caused a significant increase in twitch force under control conditions (fig. 1b; age/obesity, $\mathrm{p}=0.026$; Tempol, $\mathrm{p}=0.001$, two-way ANOVA). There was no interaction. In hypoxia, there was significantly greater specific force in aged obese muscle in comparison to young lean muscle (fig. 1d; $p<0.0001$, two-way ANOVA). There was no effect of Tempol and no interaction. Contractile kinetics for young lean and aged obese animals in control and hypoxic conditions are shown in table 1 .

\section{Peak Tetanic Force}

Both age/obesity and Tempol significantly increased the peak force (fig. $2 \mathrm{~b}$; age/obesity, $\mathrm{p}=0.006$; Tempol, $\mathrm{p}=$ 0.004, two-way ANOVA). There was no interaction. In hypoxia (fig. $2 \mathrm{~d}$ ), both age/obesity and Tempol significantly increased the force (age, $\mathrm{p}<0.0001$; Tempol, $\mathrm{p}=$ 0.023, two-way ANOVA). There was no interaction. aged obese muscle in comparison to young lean muscle. There was no effect of Tempol and no interaction. YL = Young lean; $\mathrm{AO}=$ aged obese. For control: $\mathrm{YL}, \mathrm{n}=7 ; \mathrm{YL}+\mathrm{Tempol}, \mathrm{n}=7 ; \mathrm{AO}$, $\mathrm{n}=8 ; \mathrm{AO}+$ Tempol, $\mathrm{n}=8$. For hypoxia: $\mathrm{YL}, \mathrm{n}=8 ; \mathrm{YL}+$ Tempol, $\mathrm{n}=8 ; \mathrm{AO}, \mathrm{n}=8 ; \mathrm{AO}+$ Tempol, $\mathrm{n}=8$.

\section{Force-Frequency Relationship}

Both age/obesity and Tempol caused a significant increase in force (hyperoxia, fig. $3 \mathrm{a}$ - age/obesity, $\mathrm{p}=0.001$; Tempol, $\mathrm{p}<0.001$. Hypoxia, fig. $3 \mathrm{c}$, age/obesity, $\mathrm{p}<0.001$; Tempol, $\mathrm{p}<0.001$, three-way ANOVA). In control preparations, both age/obesity and Tempol significantly reduced the $\mathrm{EF}_{50}$ (fig. $3 \mathrm{~b}$; age/obesity, $\mathrm{p}=0.001$; Tempol, $\mathrm{p}=0.001$, two-way ANOVA). There was no interaction. In hypoxia, both age/obesity and Tempol caused significant reductions in $\mathrm{EF}_{50}$ (fig. 3d; age/obesity, $\mathrm{p}<0.0001$; Tempol, $\mathrm{p}<0.0001$, two-way ANOVA). There was no significant interaction.

\section{Potentiation, Fatigue and Performance Index during \\ Repeated Muscle Stimulation}

Data illustrating sternohyoid muscle performance during repeated stimulation in young lean and aged obese rats in the absence (control) and presence of Tempol are shown for control (fig. $4 \mathrm{a}-\mathrm{d}, 5 \mathrm{a}-\mathrm{c}$ ) and hypoxic (fig. $4 \mathrm{e}-\mathrm{h}, 5 \mathrm{~d}-\mathrm{f}$ ) conditions. In the fatigue trials, both age/obesity and Tempol caused greater force production 


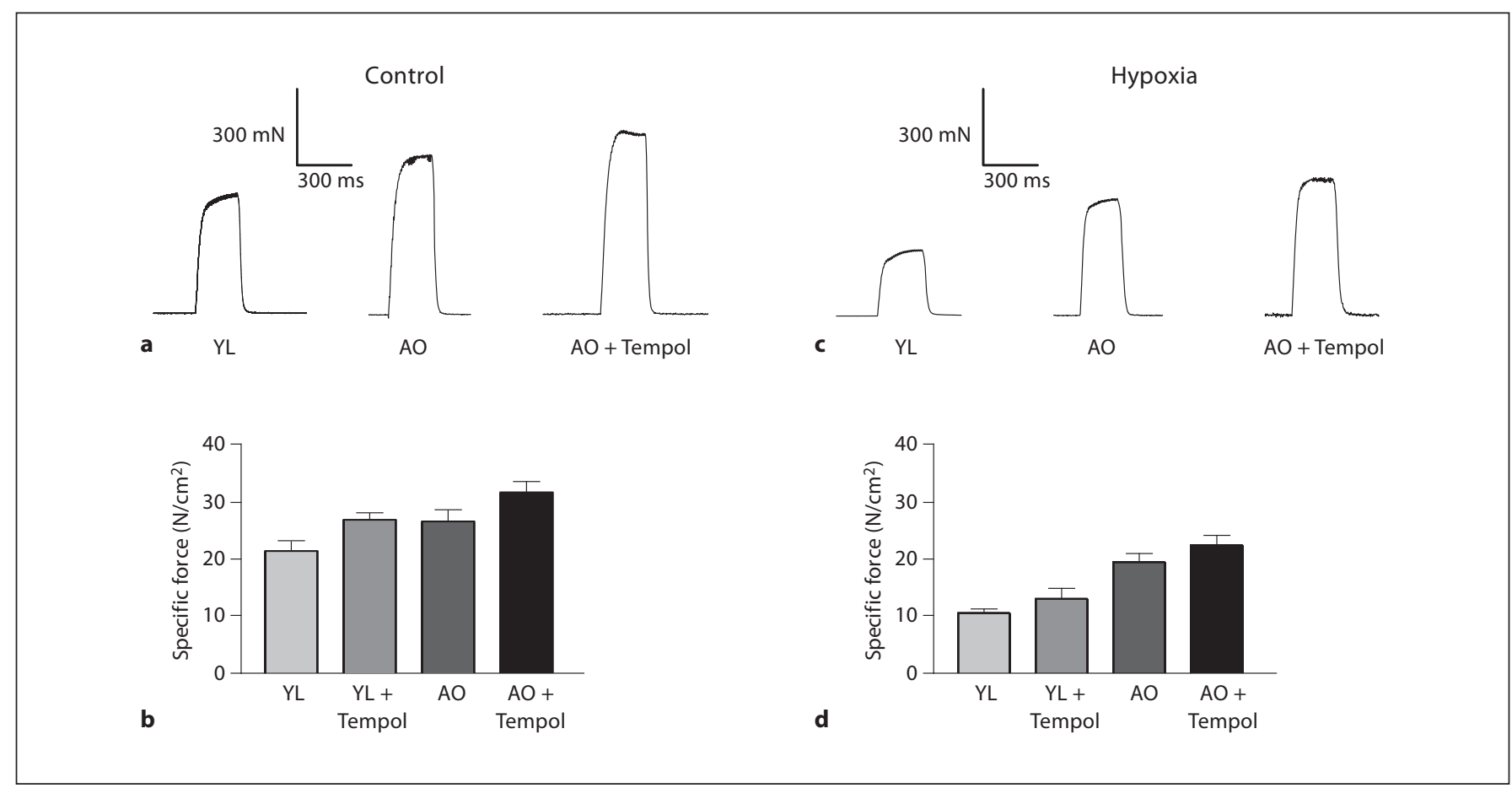

Fig. 2. Peak force. Original traces of peak force at $100 \mathrm{~Hz}$ in control (a) and hypoxic (c) conditions. b Values (means \pm SEM) for the peak tetanic force. Both age/obesity and Tempol significantly increased the peak force. There was no interaction. In hypoxia (d), both age/obesity and Tempol significantly increased the peak force. There was no interaction. Hypoxia caused a significant de- crease in force production in both young lean and aged obese muscle compared to controls. $\mathrm{YL}=$ Young lean; $\mathrm{AO}=$ aged obese. For control: YL, $\mathrm{n}=7$; YL + Tempol, $\mathrm{n}=7 ; \mathrm{AO}, \mathrm{n}=8$; $\mathrm{AO}+$ Tempol, $\mathrm{n}=8$. For hypoxia: $\mathrm{YL}, \mathrm{n}=8 ; \mathrm{YL}+$ Tempol, $\mathrm{n}=8 ; \mathrm{AO}, \mathrm{n}=8$; $\mathrm{AO}+$ Tempol, $\mathrm{n}=8$.

Table 1. Twitch kinetics of sternohyoid muscle

\begin{tabular}{|c|c|c|c|c|}
\hline Control & $\mathrm{YL}(\mathrm{n}=7)$ & $\mathrm{YL}+$ Tempol $(\mathrm{n}=7)$ & $\mathrm{AO}(\mathrm{n}=8)$ & $\mathrm{AO}+$ Tempol $(\mathrm{n}=8)$ \\
\hline Contraction time, ms & $13.3 \pm 0.3$ & $15.5 \pm 1.0^{* *}$ & $17.7 \pm 1.8^{*}$ & $19.1 \pm 2.0$ \\
\hline Half-relaxation time, ms & $9.9 \pm 1.6$ & $12.2 \pm 2.7$ & $13.1 \pm 1.7$ & $17.3 \pm 2.4$ \\
\hline Hypoxia & $\mathrm{YL}(\mathrm{n}=8)$ & $\mathrm{YL}+$ Tempol $(\mathrm{n}=8)$ & $\mathrm{AO}(\mathrm{n}=8)$ & $\mathrm{AO}+$ Tempol $(\mathrm{n}=8)$ \\
\hline $\begin{array}{l}\text { Contraction time, ms } \\
\text { Half-relaxation time, ms }\end{array}$ & $\begin{array}{r}13.6 \pm 0.1 \\
8.1 \pm 0.3\end{array}$ & $\begin{array}{r}13.0 \pm 0.3 \\
8.5 \pm 0.4\end{array}$ & $\begin{array}{l}14.5 \pm 0.5 \\
10.2 \pm 0.7^{* * *}\end{array}$ & $\begin{array}{l}15.9 \pm 0.4^{* * *} \\
10.5 \pm 0.7\end{array}$ \\
\hline
\end{tabular}

Values (means $\pm \mathrm{SEM}$ ) for single twitch kinetics of sternohyoid muscle in control and hypoxic conditions. Data is shown for young lean and aged obese groups in drug-free Krebs solution or Krebs containing $10 \mathrm{mM}$ Tempol. Under control conditions, the contraction time was increased in aged obese muscle compared to young lean muscle ( ${ }^{*} \mathrm{p}<0.001$, Mann-Whitney test). Tempol sig- nificantly increased the contraction time in young lean sternohyoid muscle $\left({ }^{* *} \mathrm{p}<0.01\right.$, Mann-Whitney test). In hypoxia, the halfrelaxation time was increased in aged obese muscle in comparison to young lean muscle $\left({ }^{* * *} \mathrm{p}<0.05\right.$, Mann-Whitney test). Tempol increased the contraction time in aged obese sternohyoid ${ }^{* * *} \mathrm{p}<$ 0.05, Mann-Whitney test). $\mathrm{YL}=$ Young lean; $\mathrm{AO}=$ aged obese. 


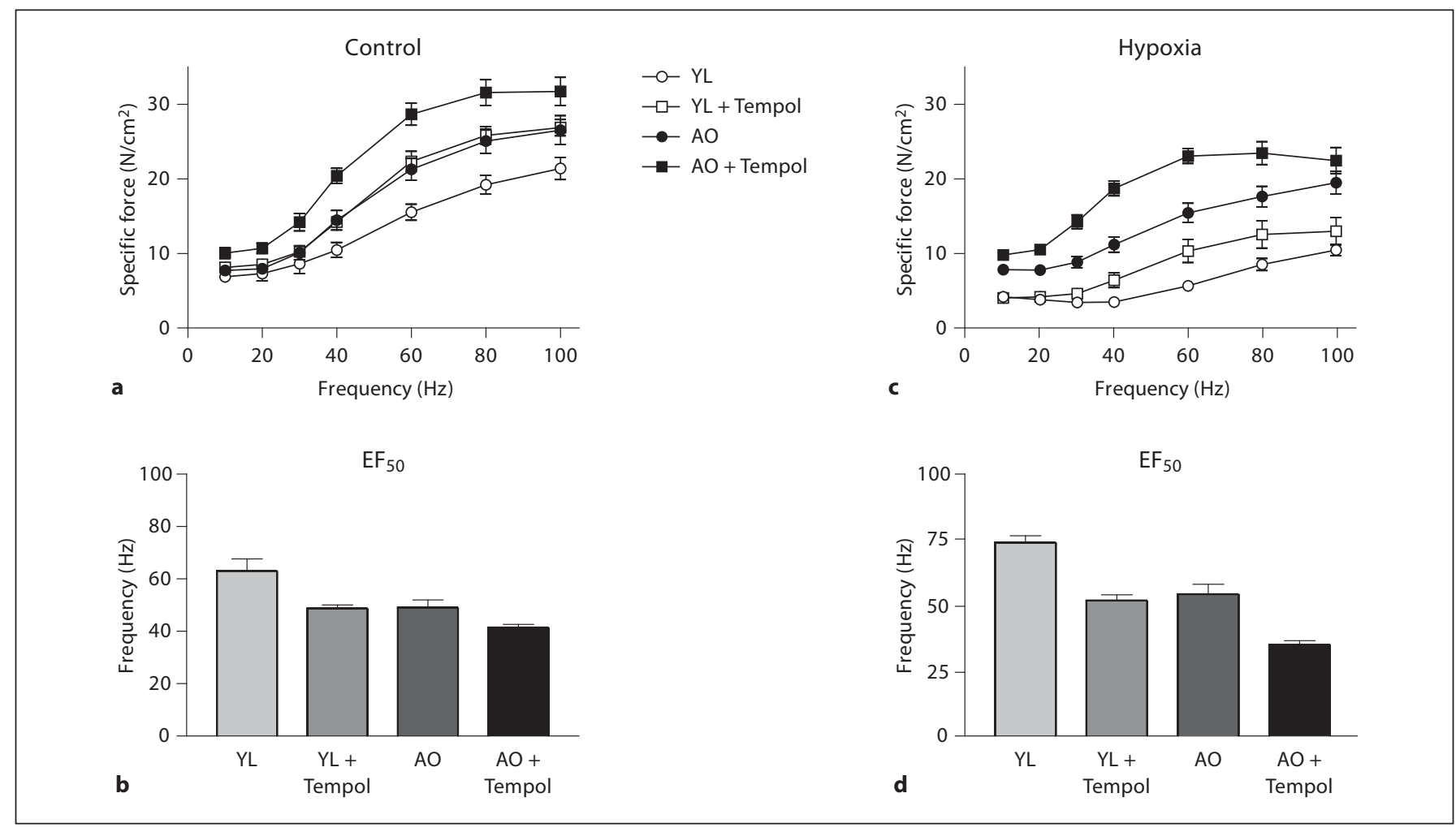

Fig. 3. Force-frequency relationship. a Group values (means \pm SEM) for the force-frequency relationship under control conditions. There was a significant increase in force for both the aged obese and Tempol groups. b Both age/obesity and Tempol significantly reduced $\mathrm{EF}_{50}$. c Group values (means $\pm \mathrm{SEM}$ ) for the force-frequency relationship under control conditions. There was a significant increase in force for both the aged obese and Tempol groups. $\mathbf{d}$ Both age/obesity and Tempol caused significant reductions in $\mathrm{EF}_{50}$ in hypoxia. $\mathrm{YL}=$ Young lean; $\mathrm{AO}=$ aged obese. For control: YL, $\mathrm{n}=7$; YL + Tempol, $\mathrm{n}=7 ; \mathrm{AO}, \mathrm{n}=8$; $\mathrm{AO}+$ Tempol, $\mathrm{n}=8$. For hypoxia: YL, $\mathrm{n}=8$; YL + Tempol, $\mathrm{n}=8$; AO, $\mathrm{n}=8$; $\mathrm{AO}+$ Tempol, $\mathrm{n}=8$. in control and hypoxic conditions (control, fig. $4 \mathrm{~d}-$ age/ obesity, $\mathrm{p}=0.003$; Tempol, $\mathrm{p}<0.001$; time, $\mathrm{p}<0.001$. Hypoxia, fig. $4 \mathrm{~h}-$ age/obesity, $\mathrm{p}<0.001$; Tempol, $\mathrm{p}<$ 0.001 , three-way ANOVA). In hypoxia, there was an enhanced positive inotropic effect of Tempol in the aged obese tissue in comparison to that of young lean muscle early in the hypoxic fatigue trial [interaction (age/obesity $\times$ Tempol $\times$ time), $p=0.002$, three-way ANOVA]. Force potentiation in response to repeated stimulation was observed in all sternohyoid preparations from young lean and aged obese rats and the magnitude of the effect was similar in the two groups in control preparations (fig. 5a). In hypoxia (fig. 5d), Tempol decreased potentiation in the aged obese group but not in the young lean group [interaction (age/obesity $\times$ Tempol), $\mathrm{p}=0.001$, two-way ANOVA].

Under control conditions (fig. 5b), Tempol increased the fatigue index (Tempol, $\mathrm{p}<0.0001$, two-way ANOVA).
There was no effect of age/obesity and no interaction. The performance index of the sternohyoid was unaffected by either age/obesity or the drug (fig. 5c). In hypoxia (fig. 5e), both age/obesity and Tempol increased the fatigue index (age, $\mathrm{p}<0.0001$; Tempol, $\mathrm{p}=0.0002$, two-way ANOVA). Tempol significantly decreased the performance index in aged obese animals [fig. 5f; age, $\mathrm{p}=0.034$; Tempol, $\mathrm{p}<$ 0.0001 ; interaction (age/obesity $\times$ Tempol), $\mathrm{p}=0.0071$, two-way ANOVA].

\section{MHC Composition, Fibre Morphometry, SDH and \\ GPDH Enzyme Activities and TBARS Assay}

Representative MHC immunofluorescence images from young lean and aged obese animals are shown in figure $6 \mathrm{a}, \mathrm{b}$. Group data in table 2 shows that the areal density for type 2A fibres was significantly decreased $(\mathrm{p}<0.001$, one-way ANOVA), whereas the areal density for type $2 \mathrm{X}$ fibres was significantly increased $(\mathrm{p}<0.001$, 


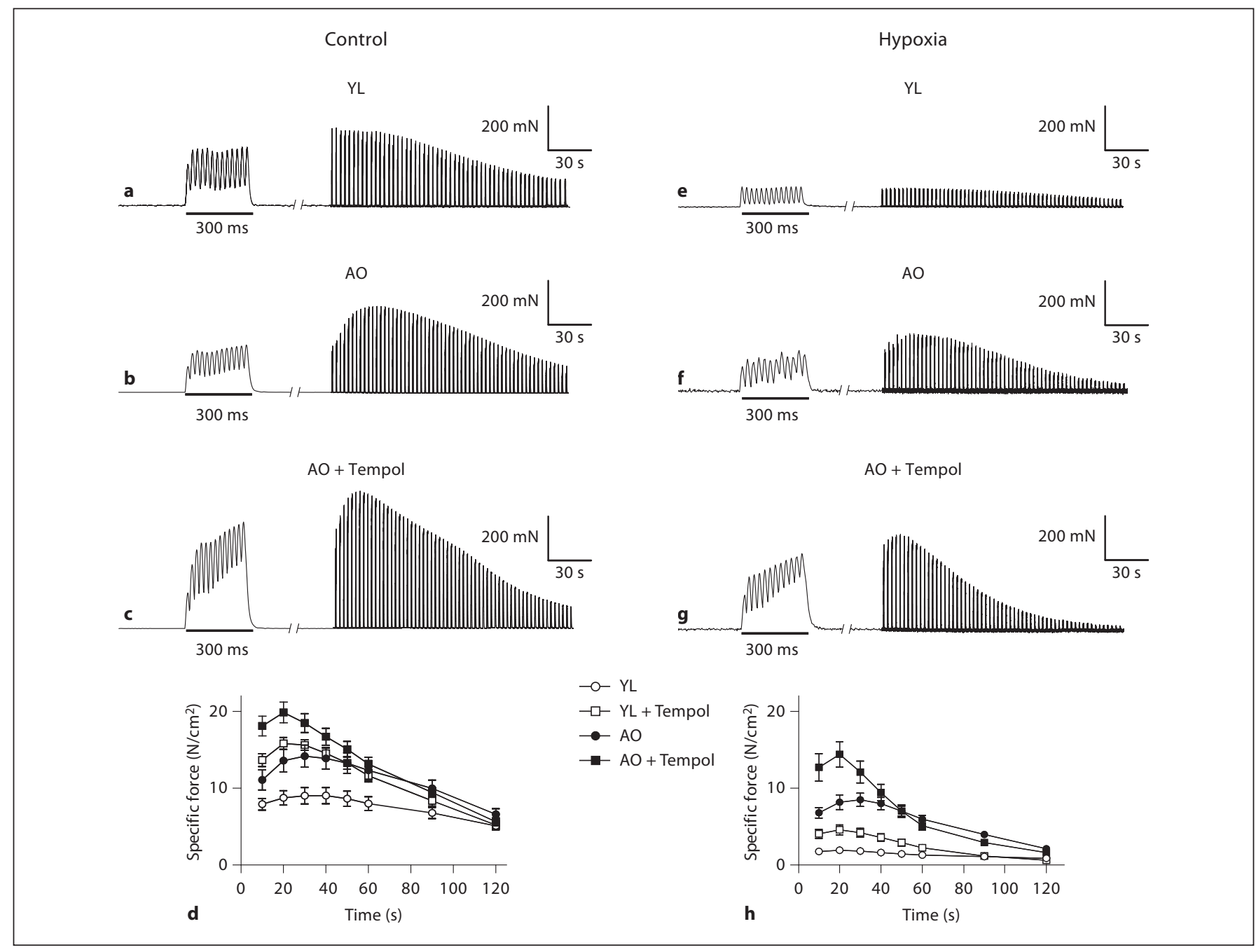

Fig. 4. Repeated stimulation. a-c Original traces of repeated muscle stimulation under control conditions. d Both age/obesity and Tempol had positive inotropic effects on force production throughout the fatigue trial. e-g Original traces of repeated muscle stimulation in hypoxia. $\mathbf{h}$ Both age/obesity and Tempol had positive inotropic effects on force production throughout the fa-

one-way ANOVA) in aged obese muscles in comparison to young lean muscles. Hypertrophy of all four fibre types was observed in aged obese sternohyoid ( $p<0.001$, oneway ANOVA). SDH activity was significantly decreased ( $\mathrm{p}<0.05$, Student's unpaired t test) and GPDH activity was significantly increased $(\mathrm{p}<0.05$, Student's unpaired $t$ test) in aged obese muscle. TBARS levels were not significantly different in young lean and aged obese sternohyoid muscle $(0.64 \pm 0.18$ vs. $0.54 \pm 0.16 \mathrm{nmol} \mathrm{MDA} / \mathrm{mg}$ protein, young vs. aged, $\mathrm{p}=0.67$, Student's unpaired $\mathrm{t}$ test). tigue trial. Note that the effect of Tempol was enhanced in aged obese tissue in comparison to that of young lean muscle. $\mathrm{YL}=$ Young lean; $\mathrm{AO}=$ aged obese. For control: $\mathrm{YL}, \mathrm{n}=7 ; \mathrm{YL}+$ Tempol, $\mathrm{n}=7 ; \mathrm{AO}, \mathrm{n}=8 ; \mathrm{AO}+$ Tempol, $\mathrm{n}=7$. For hypoxia: $\mathrm{YL}, \mathrm{n}=6$; $\mathrm{YL}+$ Tempol, $\mathrm{n}=8 ; \mathrm{AO}, \mathrm{n}=8 ; \mathrm{AO}+$ Tempol, $\mathrm{n}=8$.

\section{Discussion}

The findings of the present study show that the muscle fibre types of the sternohyoid in aged obese rat are larger and stronger compared to the young lean rat and, surprisingly, this old airway muscle is very resilient to oxidative stress. Our original hypothesis that the aged obese muscle, despite its indeterminate growth pattern, would exhibit diminished performance especially when subjected to oxidative stress is rejected. 
Fig. 5. Potentiation, fatigue index and performance. Group data (means \pm SEM) for force potentiation during repeated muscle stimulation trials under control (a) and hypoxic (d) conditions. In hypoxia (d), Tempol decreased force potentiation in the aged obese group. Group data (means \pm SEM) for fatigue index during repeated muscle stimulation trials under control (b) and hypoxic (e) conditions. In control conditions (c), Tempol increased the fatigue index. In hypoxia (e), both age/obesity and Tempol increased the fatigue index. Group data (means \pm SEM) for the performance index during the repeated muscle stimulation protocol in control (c) and hypoxic (f) conditions. The performance index was unaffected by either age/obesity or Tempol (c). In hypoxia (f), Tempol significantly decreased the performance index in the aged obese group. $\mathrm{YL}=$ Young lean; $\mathrm{AO}=$ aged obese. For control: YL, n = 7; YL + Tempol, $\mathrm{n}=7 ; \mathrm{AO}, \mathrm{n}=8 ; \mathrm{AO}+$ Tempol, $\mathrm{n}=7$. For hypoxia: YL, $\mathrm{n}=6$; YL + Tempol, $\mathrm{n}=8$; $\mathrm{AO}, \mathrm{n}=8 ; \mathrm{AO}+$ Tempol, $\mathrm{n}=8)$.
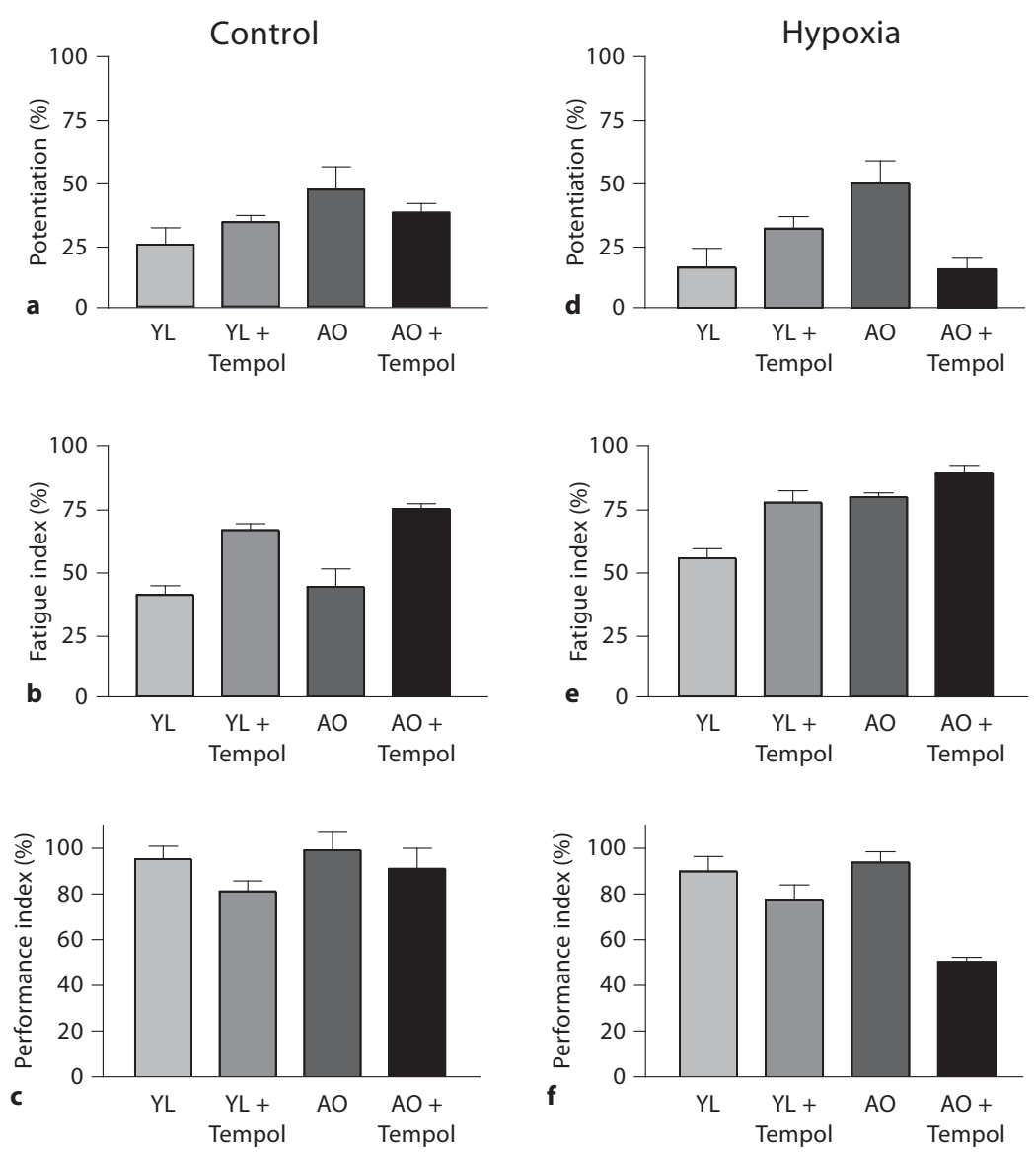
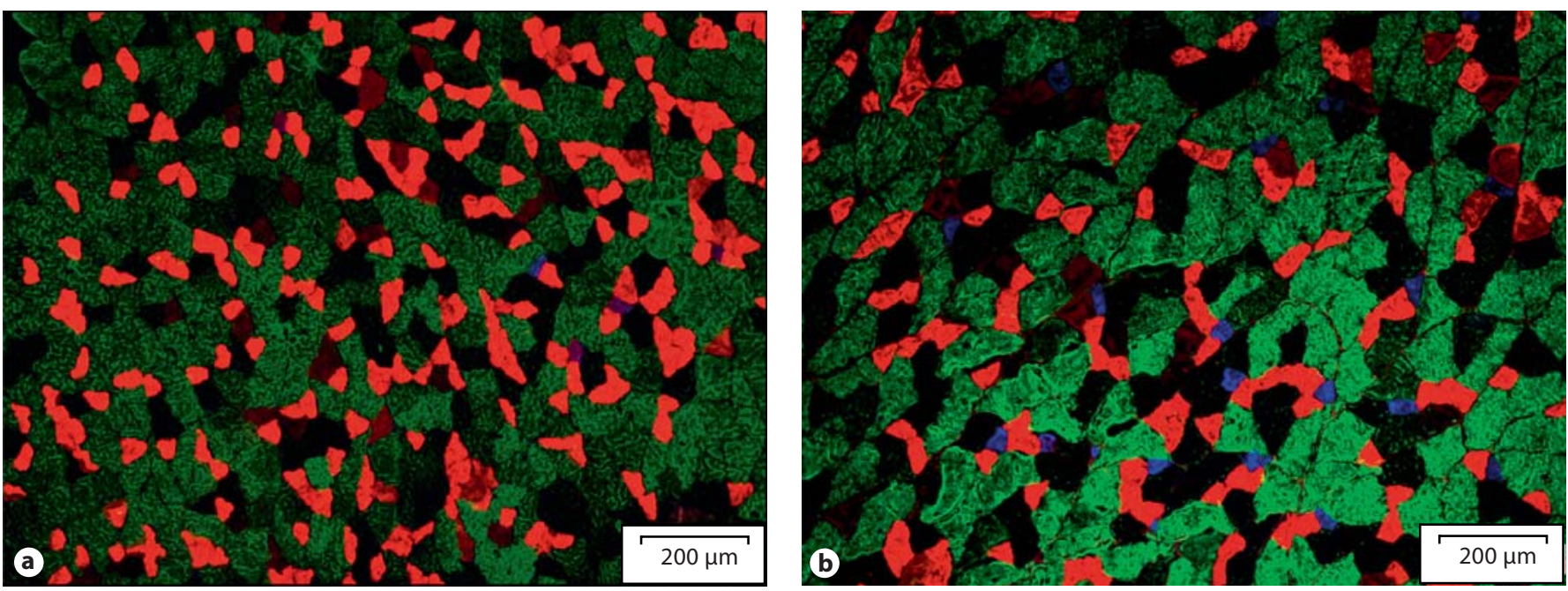

Fig. 6. Immunohistochemistry. Representative triple-labelled (merged image) immunofluorescent image of young lean (a) and aged obese (b) sternohyoid muscle showing MHC type 1 (blue), 2A (red), 2B (green) and 2X (unmarked) fibres. 
The present study highlights the structural and biochemical adjustments in aged obese sternohyoid muscle which would appear to contribute to increased force and hypoxic tolerance. Fibre type transformation (oxidative to glycolytic) and decreased SDH activity were seen in aged obese sternohyoid muscle, consistent with previous reports for this muscle in aged rats $[5,9]$ and other aged upper airway muscles [10], while increased GPDH activity was observed in keeping with a previous report in aged rat [5]. Interestingly, there was no evidence of increased lipid peroxidation (a marker of oxidative stress in tissues) in aged obese sternohyoid muscle, though this has been reported in limb muscle of older rats [11, 12]. Furthermore, fibre hypertrophy and a $2 \mathrm{~A}$-to-2X fibre transition were observed in aged obese airway muscle. This is of particular interest because slow-to-fast fibre transitions and fibre hypertrophy in upper airway muscles are reported in OSAS patients [13] and in the English bulldog [14] which is an excellent animal model of OSAS.

Areal densities of fibres were calculated to vividly convey the contribution of each fibre type to specific force production. The data of table 2 also permit estimation of the sarcolemmal surface area per cubic millimetre of whole muscle or specific fibre types. We determined that age is associated with a decline in total muscle fibre surface area of approximately $20 \%$ from 61,829 to 49,088 $\mu \mathrm{m}^{2} \cdot \mathrm{mm}^{-3}$. This decline is due to a fibre transition from $2 \mathrm{~A}$ to $2 \mathrm{X}$. This age-related decline in sarcolemmal surface area, however, is not associated with a decline in muscle performance; indeed we observed increased muscle-specific force in keeping with the increase in fibre cross-sectional area. Surface area-to-volume adjustment in muscle is a potential allometric trap for investigators. All proteins associated with the membrane surface (ion channels, pumps, exchangers) would also show a change when expressed as a value per unit mass of muscle or protein (under conditions of constant membrane density).

We observed positive inotropy and a significant left shift in the force-frequency relationship of aged obese muscle, as evidenced by a decrease in $\mathrm{EF}_{50}$. This suggests that aged obese upper airway muscle has increased sensitivity to electrical stimulation. Given that the sternohyoid is an important airway dilator $[15,16]$, we speculate that structural and functional remodelling in aged obese airway dilator muscle is a beneficial 'adaptation' with implications for the control of airway patency. It is possible that the physiological adaptations in the sternohyoid muscle are compensatory in nature, resulting for example from increased respiratory muscle loading or perhaps alterations in non-respiratory functions of the hyoid muscles
Table 2. Sternohyoid muscle fibre areal density and metabolic activities

$$
\mathrm{YL}(\mathrm{n}=7) \quad \mathrm{AO}(\mathrm{n}=6)
$$

Immunohistochemistry

Areal density, \%

$\mathrm{MHC}$

$\begin{array}{lrc}1 & 1.1 \pm 0.3 & 1.4 \pm 0.3 \\ 2 \mathrm{~A} & 21.2 \pm 0.9 & 14.1 \pm 1.0^{* *} \\ 2 \mathrm{X} & 7.9 \pm 0.6 & 18.0 \pm 1.1^{* *} \\ 2 \mathrm{~B} & 65.5 \pm 1.3 & 62.4 \pm 1.9\end{array}$

Cross-sectional area, $\mu \mathrm{m}^{2}$ MHC

1

$2 \mathrm{~A}$

$2 \mathrm{X}$

$627 \pm 40$

$1,286 \pm 79^{* * *}$

$1,353 \pm 123$

$2,190 \pm 126^{* * * *}$

$2 \mathrm{~B}$

$1,807 \pm 109$

$3,043 \pm 195^{* *}$

$6,863 \pm 2,048^{* *}$

Enzyme histochemistry optical density, $A U$
$\mathrm{SDH}$
$65.6 \pm 5.1$
$49.0 \pm 3.4^{* *}$
GPDH
$108.4 \pm 1.9$
$121.2 \pm 2.1^{* *}$

Values (means \pm SEM) for muscle fibre areal density. Note the significant decrease in type $2 \mathrm{~A}$ and increase in type $2 \mathrm{X}$ areal density in aged obese sternohyoid. Values (means \pm SEM) for muscle fibre cross-sectional area. There was a significant increase in all fibre cross-sectional areas in aged obese muscles compared to young lean muscles. SDH activity was significantly decreased in aged obese muscle compared to young lean muscle. GPDH activity was significantly increased in aged obese muscle compared to young lean muscle. $\mathrm{YL}=$ Young lean; $\mathrm{AO}=$ aged obese.

Significantly different from the corresponding control: ${ }^{* *} \mathrm{p}<$ $0.01 ;{ }^{* * *} \mathrm{p}<0.05$.

in aged obese animals. Alternatively, the changes could merely reflect the natural development and aging process in the rat which would appear to provide protection to this airway dilator muscle. The mechanisms that underpin these changes remain unclear.

Since ROS are implicated in skeletal muscle function and antioxidants have been shown to improve skeletal muscle performance $[17,18]$ including diaphragm [19] and pharyngeal dilator [20] muscle function, we sought to test the effects of an antioxidant on sternohyoid muscle performance in aged obese rats. Our results are consistent with and extend our previous findings in young lean airway muscle [20]. We believed that it was important, however, to assess the efficacy of Tempol in aged obese muscle since previous studies have demonstrated that aging negates the beneficial effects of almitrine [21,22] and a potassium channel blocker [23] on respiratory muscle function. In aged obese muscle we observed a positive inotropic effect of Tempol, which was maintained during 
repeated muscle activation. The superoxide scavenger was more effective in aged obese muscle compared to young lean muscle during hypoxia, with evidence of increased sensitivity to stimulation (i.e. a decrease in $\mathrm{EF}_{50}$ ) and increased tetanic force. Tempol dramatically rescued force in hypoxia, consistent with observations that superoxide scavengers protect diaphragm muscle contractile function in severe hypoxia $[19,24]$.

Pharmacological therapy has been suggested as a clinical strategy in the treatment of OSAS $[25,26]$ and agents that improve upper airway muscle performance could serve as viable adjunct therapies for patients with sleepdisordered breathing. The effects of Tempol reported herein are likely to be beneficial in terms of the maintenance and/or restoration of airway calibre, assuming a similar action of the drug in humans. Increased upper airway muscle sensitivity could potentially help to offset the natural sleep-related decrements in cranial motor drive, and increased force of contraction in response to high stimulus rates could facilitate airway re-opening following occlusion. Whilst we acknowledge that Tempol may be ineffective in preventing airway collapse during REM sleep, when motor drive to some airway muscles is negligible the drug and similar compounds could serve to expedite recovery of a collapsed airway segment and in this way limit the duration and severity of apnoeic events. Moreover, as OSAS is now widely recognized as an oxidative stress disorder $[27,28]$, antioxidant supplementation is also likely to limit oxidative stress associated with recurrent hypoxaemia - a hallmark feature of sleep-disordered breathing. This is important when one considers that chronic intermittent hypoxia-induced oxidative injury may well be the major contributor to the development of key morbidities associated with OSAS [28].

There are several significant limitations to this study. First, we examined only one airway muscle - the sterno- hyoid - which we assume is generally representative of upper airway muscles, but other pharyngeal dilators such as the genioglossus - pivotal in the control and maintenance of upper airway patency - were not studied. The sternohyoid muscle was chosen for study because it is an important airway dilator muscle $[15,16]$ which shows evidence of remodelling in the English bulldog - a natural model of OSAS [14]. Also (unlike the genioglossus), the muscle is readily accessible and its fibres are arranged longitudinally which makes it ideal for in vitro assessment of contractile function. Our conclusions are based on data derived from an isolated muscle preparation which are most likely reflective of sternohyoid muscle performance in vivo, but this is a major assumption. Finally, our study employed an animal model and we recognize the need to exercise caution in extrapolating the data to human subjects.

To conclude, the indeterminate growth pattern of the rat sternohyoid muscle is associated with a protective action against oxidative stress. With regard to human OSAS, it is of interest that the muscle changes occurring in the disorder resemble the natural programme of aging in the rat (muscle hypertrophy, fibre and metabolic transitions). Our results may have relevance for the control of airway patency in old obese subjects.

\section{Acknowledgments}

This study was funded by the Health Research Board Ireland (grants RP/2006/140 and RP/2008/159 to K.D. O'Halloran). J.R. Skelly is a UCD Ad Astra Research Scholar. R.A. O'Connell is funded by the Irish Research Council for Science, Engineering and Technology. We are grateful to Clodagh McMorrow, PhD, for advice on immunohistochemical protocols and to Stephen Fahey, $\mathrm{BSc}$, for performing the lipid peroxidation assay.

\section{References}

1 Roach HI, Mehta G, Oreffo RO, Clarke NM, Cooper C: Temporal analysis of rat growth plates: cessation of growth with age despite presence of a physis. J Histochem Cytochem 2003;51:373-383.

2 Verbraecken JA, De Backer WA: Upper airway mechanics. Respiration 2009;78:121-133.

-3 Palla A, Digiorgio M, Carpene N, Rossi G, D’Amico I, Santini F, Pinchera A: Sleep apnea in morbidly obese patients: prevalence and clinical predictivity. Respiration 2009; 78:134-140.
4 Van Lunteren E, Vafaie H, Salomone RJ: Comparative effects of aging on pharyngeal and diaphragm muscles. Respir Physiol 1995;99:113-125.

5 Ray AD, Magalang UJ, Michlin CP, Ogasa T, Krasney JA, Gosselin LE, Farkas GA: Intermittent hypoxia reduces upper airway stability in lean but not obese Zucker rats. Am J Physiol Regul Integr Comp Physiol 2007; 293:R372-R378.
6 Van Lunteren E, Vafaie H, Miller MJ: Effects of theophylline on pharyngeal dilator and diaphragm muscle contractile properties. Respiration 1996;63:88-93.

7 Punkt K, Zaitsev, S, Park JK, Wellner M, Buchwalow IB: Nitric oxide synthase isoforms I, III and protein kinase-Ctheta in skeletal muscle fibres of normal and streptozotocin-induced diabetic rats with and without Ginkgo biloba extract treatment. Histochem J 2001;33:213-219. 
$>8$ Healy CF, McMorrow C, O’Herlihy C, O'Connell PR, Jones JF: External anal sphincter fatigue is not improved by $\mathrm{N}$-acetylcysteine in an animal model. Neurogastroenterol Motil 2008;20:719-724.

$>9$ Ray AD, Ogasa T, Magalang UJ, Krasney JA, Farkas GA: Aging increases upper airway collapsibility in Fischer 344 rats. J Appl Physiol 2008;105:1471-1476.

10 Oliven A, Carmi N, Coleman R, Odeh M, Silbermann M: Age-related changes in upper airway muscles morphological and oxidative properties. Exp Gerontol 2001;36:16731686.

11 Ji LL, Dillon D, Wu E: Alteration of antioxidant enzymes with aging in rat skeletal muscle and liver. Am J Physiol 1990;258:R918R923.

-12 Ryan MJ, Dudash HJ, Docherty M, Geronilla KB, Baker BA, Haff GG, Cutlip RG, Alway SE: Aging-dependent regulation of antioxidant enzymes and redox status in chronically loaded rat dorsiflexor muscles. J Gerontol A Biol Sci Med Sci 2008;63:1015-1026.

13 Ferini-Strambi LJ, Smirne S, Moz U, Sferrazza B, Iannaccone S: Muscle fibre type and obstructive sleep apnea. Sleep Res Online 1998; 1:24-27.

14 Petrof BJ, Pack AI, Kelly AM, Eby J, Hendricks JC: Pharyngeal myopathy of loaded upper airway in dogs with sleep apnea. J Appl Physiol 1994;76:1746-1752.
15 Roberts JL, Reed WR, Thach BT: Pharyngeal airway stabilizing function of sternohyoid and sternothyroid muscles in the rabbit. J Appl Physiol 1984;57:1790-1795.

16 Van Lunteren E, Haxhiu MA, Cherniack NS: Relation between upper airway volume and hyoid muscle length. J Appl Physiol 1987;63: 1443-1449.

17 Reid MB: Free radicals and muscle fatigue: of ROS, canaries, and the IOC. Free Radic Biol Med 2008;44:169-179.

18 Reid MB, Haack KE, Franchek KM, Valberg PA, Kobzik L, West MS: Reactive oxygen in skeletal muscle. 1. Intracellular oxidant kinetics and fatigue in vitro. J Appl Physiol 1992;73:1797-1804.

19 Wright VP, Klawitter PF, Iscru DF, Merola AJ, Clanton TL: Superoxide scavengers augment contractile but not energetic responses to hypoxia in rat diaphragm. J Appl Physiol 2005;98:1753-1760.

20 Skelly JR, Bradford A, Jones JF, O’Halloran KD: Superoxide scavengers improve rat pharyngeal dilator muscle performance. Am J Respir Cell Mol Biol 2010;42:725-731.
21 Cantillon D, Bradford A: Effect of almitrine on upper airway muscle contraction in young and old rats. Eur J Pharmacol 2001; 412:187-194.

22 McGuire M, Cantillon D, Bradford A: Effects of almitrine on diaphragm contractile properties in young and old rats. Respiration 2002;69:75-80.

23 Van Lunteren E, Moyer M: Electrophysiologic and inotropic effects of $\mathrm{K}^{+}$-channel blockade in aged diaphragm. Am J Respir Crit Care Med 1998;158:820-826.

24 Mohanraj P, Merola AJ, Wright VP, Clanton TL: Antioxidants protect rat diaphragmatic muscle function under hypoxic conditions. J Appl Physiol 1998;84:1960-1966.

25 Hudgel DW, Thanakitcharu S: Pharmacologic treatment of sleep-disordered breathing. Am J Respir Crit Care Med 1998;158: 691-699.

26 Hedner J, Grote L, Zou D: Pharmacological treatment of sleep apnea: current situation and future strategies. Sleep Med Rev 2008; 12:33-47.

27 Lavie L: Obstructive sleep apnoea syndrome - an oxidative stress disorder. Sleep Med Rev 2003;7:35-51.

28 Lavie L: Oxidative stress - a unifying paradigm in obstructive sleep apnea and comorbidities. Prog Cardiovasc Dis 2009;51:303312. 